\title{
TRATAMENTO ARTROSCÓPICO DA LUXAÇÃO ACROMIO-CLAVICULAR PELO MÉTODO "TIGHT ROPE" (ARTHREX ${ }^{\circledR}$ )
}

\section{ARTHROSCOPIC TREATMENT OF ACROMIOCLAVICULAR JOINT DISLOCATION BY TIGHT ROPE TECHNIQUE (ARTHREX')}

\author{
Luis Alfredo Gómez Vieira', \\ Adalberto Visco², \\ Luis Filipe Daneu Fernandes ${ }^{3}$, \\ Nicolas Gerardo Gómez Cordero 4
}

\section{RESUMO}

Objetivo: Apresentar a técnica cirúrgica artroscópica pelo método "Tight Rope" e a avaliação dos resultados com esta técnica no tratamento da luxação acrômio-clavicular aguda. Métodos: entre agosto de 2006 e maio de 2007, 10 ombros de 10 pacientes com luxação acrômio-clavicular aguda foram submetidos a tratamento artroscópcio pela técnica Tight Rope-Arthrex ${ }^{\circledR}$. O seguimento mínimo foi de 12 meses, com média de 15 meses. A idade variou de 26 e 42 anos com média de 34 anos. Todos os pacientes eram do sexo masculino. Todos os pacientes foram atendidos na fase aguda da lesão sendo avaliados por radiologia simples (série trauma). Os pacientes foram acompanhados semanalmente no primeiro mês e a cada três meses após o procedimento artroscópico. A avaliação clínica foi feita por meio dos critérios da University of Califórnia at Los Angeles (UCLA). Resultados: Todos os pacientes operados agudamente encontravam-se satisfeitos com os resultados do tratamento cirúrgico artroscópico com uma média de 32,5 pontos na escala de avaliação da UCLA. Conclusão: O tratamento artroscópico da luxação acrômio-clavicular aguda pelo método "Tight Rope" é uma técnica cirúrgica minimamente invasiva que mostrou-se eficiente para o tratamento destas lesões.

Descritores - Luxação acrômio-clavicular/cirurgia; Artroscopia/métodos

\begin{abstract}
Objective: Presenting the arthroscopic treatment by Tight Rope - Arthrex ${ }^{\circledR}$ system for acute acromioclavicular dislocation and to evaluate results obtained with this procedure. Methods: Between August 2006 and May 2007, 10 shoulders of 10 patients with acute acromioclavicular dislocation were submitted to arthroscopic repair using the Tight Rope - Arthrex ${ }^{\circledR}$ system. Minimum follow-up was 12 months, with a mean of 15 months. Age ranged from 26 to 42, mean 34 years. All patients were male. Radiology evaluation was made by trauma series $x$-ray. The patients were assisted in the first month weekly and after three months after the procedure. Clinical evaluation was based on the University of California at Los Angeles (UCLA) criteria. Results: All patients were satisfied after the arthroscopic procedure and the mean UCLA score was 32,5. Conclusion: The arthroscopic treatment by Tight Rope - Arthrex ${ }^{\circledR}$ system for acute acromioclavicular dislocation showed to be an efficient technique.
\end{abstract}

Keywords - Acromioclavicular dislocation/surgery; Arthroscopy/methods

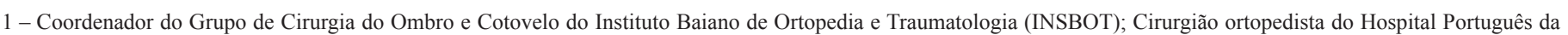
Bahia

2 - Chefe do Grupo de Cirurgia do Ombro e Cotovelo da Clínica de Fraturas ORTOPED; Cirurgião ortopedista do Hospital Português da Bahia

3 - Médico-Assistente dos Grupos de Cirurgia do Ombro e Cotovelo do Instituto Baiano de Ortopedia e Traumatologia (INSBOT) e Clínica de Fraturas ORTOPED

4 - Chefe do Serviço de Cirurgia Ortopédica do Instituto Baiano de Ortopedia e Traumatologia (INSBOT); Cirurgião ortopedista do Hospital Português da Bahia
}

Trabalho realizado no Instituto Baiano de Ortopedia e Traumatologia - INSBOT e Hospital Português da Bahia - Salvador - Bahia - Brasil

Correspondência: Avenida Anita Garibaldi, 1133, Ondina, CEP: 40210-070, Salvador -Bahia - Brasil. E-mail: gomezvieira@hotmail.com 


\section{INTRODUÇÃO}

A luxação acrômio-clavicular (LAC) representa uma das mais frequentes lesões do ombro vista na prática ortopédica geral. O mecanismo de trauma mais comum desta lesão é a queda com força direta na região lateral do ombro com o membro superior em posição de adução ${ }^{(1)}$.

Dependendo da magnitude do trauma, esta lesão pode ser classificada em seis tipos ${ }^{(2)}$. Tipicamente, os tipos I e II são tratados conservadoramente com a maioria dos pacientes retornando às condições pré-lesão ${ }^{(3-6)}$. Embora o tratamento das lesões do tipo III seja controverso, as lesões de alta energia (tipos IV, V, VI) com mais de $100 \%$ de deslocamento posterior e/ou inferior são tratadas cirurgicamente ${ }^{(7-10)}$.

Várias técnicas cirúrgicas foram desenvolvidas buscando o tratamento da luxação acrômio-clavicular ${ }^{(9,10)}$. A técnica de Weaver-Dunn que transfere o ligamento coracoacromial para o canal intramedular da clavícula distal tem sido um dos procedimentos mais comumente utilizados tanto para os casos agudos como também para os casos crônicos ${ }^{(11)}$.

O propósito deste estudo é avaliar os resultados obtidos com a técnica cirúrgica artroscópica no tratamento da luxação acromio-clavicular aguda pelo método "Tight Rope" - Arthrex ${ }^{\circledR}$.

\section{MÉTODOS}

No período de agosto de 2006 a maio de 2007 foram operados e reavaliados 10 ombros de 10 pacientes submetidos a tratamento artroscópico da LAC aguda pela técnica Tight Rope-Arhtrex ${ }^{\circledR}$. As cirurgias foram realizadas pelo Grupo de Cirurgia de Ombro e Cotovelo do Instituto Baiano de Ortopedia e Traumatologia (INSBOT) e no Hospital Português da Bahia e avaliadas após aprovação pelos Comitês de Ética das instituições.

O tempo de seguimento variou de 12 a 19 meses, com média de 15 meses. A média de idade foi de 34 anos, variando de 26 a 42 anos. Todos os pacientes eram do sexo masculino (Tabela 1).

Dor e deformidade foram encontrados em todos os pacientes deste estudo ( $100 \%$ dos casos) e o sinal da tecla esteve presente em nove pacientes (tipos IV e V).

Todos os pacientes foram atendidos na fase aguda da lesão sendo avaliados por radiologia simples (série trauma). Observou-se, com esse exame, que três pacientes apresentavam LAC tipo $\mathrm{V}$, cinco pacientes apresentavam LAC tipo IV e dois pacientes apresentava LAC tipo III. Nenhum dos pacientes costumava realizar atividades físicas regularmente. O tempo de imobilização
Tabela 1 - Dados dos pacientes

\begin{tabular}{c|c|c|c|c}
\hline $\begin{array}{c}\text { Número do } \\
\text { Paciente }\end{array}$ & Sexo & Idade & UCLA & $\begin{array}{c}\text { Seguimento } \\
\text { pós-op (meses) }\end{array}$ \\
\hline 1 & M & 36 anos & 35 & 19 \\
2 & M & 42 anos & 29 & 12 \\
3 & M & 30 anos & 29 & 15 \\
4 & M & 26 anos & 34 & 15 \\
5 & F & 34 anos & 35 & 19 \\
6 & M & 32 anos & 35 & 15 \\
7 & M & 28 anos & 30 & 15 \\
8 & M & 30 anos & 34 & 17 \\
9 & M & 34 anos & 29 & 14 \\
10 & M & 33 anos & 35 & 13 \\
\hline
\end{tabular}

Fonte: DOT-INSBOT

UCLA = University of Califórnia at Los Angeles - método de avaliação dos resultados pós-operatórios.

no período pós-operatório foi de duas semanas em todos os casos sendo, então, os pacientes encaminhados para procedimentos fisioterápicos e liberados para atividades de vida diária. $\mathrm{O}$ retorno à prática esportiva foi consentido após três meses do ato cirúrgico. Os pacientes foram acompanhados semanalmente no primeiro mês, a cada mês nos primeiros três meses e a cada três meses após o quarto mês do procedimento artroscópico.

O método escolhido para avaliação clínica dos pacientes no período pós-operatório baseou-se nos critérios da UCLA (University of Califórnia at Los Angeles) ${ }^{(12)}$.

\section{TÉCNICA CIRÚRGICA}

A cirurgia foi realizada em todos os casos com o paciente em posição "cadeira de praia". Através do portal posterior, é introduzida a óptica para realização da inspeção articular do ombro. É feito uma incisão de 1,5 $\mathrm{cm}$ sobre a clavícula ao nível do processo coracóide sob visão direta (Figura 1). Nesse momento, é colocado um guia tipo estribo sob o processo coracóide (seta maior) e sobre a clavícula através do portal anterior e na incisão clavicular sendo criados os túneis (setas menores) na clavícula e no processo coracóide (Figura 2). É, então, passado através destes túneis de superior para inferior um fio-guia maleável (seta maior) que é capturado através do portal anterior por uma pinça (seta menor) tipo grasper (Figura 3). O sistema Tight Rope-Arthrex ${ }^{\circledR}$, formado por dois botões metálicos unidos por quatro segmentos de um mesmo fio Fiberwire-Arthrex ${ }^{\circledR}$ No.5 (Figura 4), é acoplado na extremidade superior do fio-guia maleável que é puxado através do portal anterior passando um botão metálico subcoracóide (setas) enquanto que um outro botão é ajustado sob tensão sobre a clavícula (setas) após redução da articulação acrômio-clavicular (Figuras 5 e 6). 


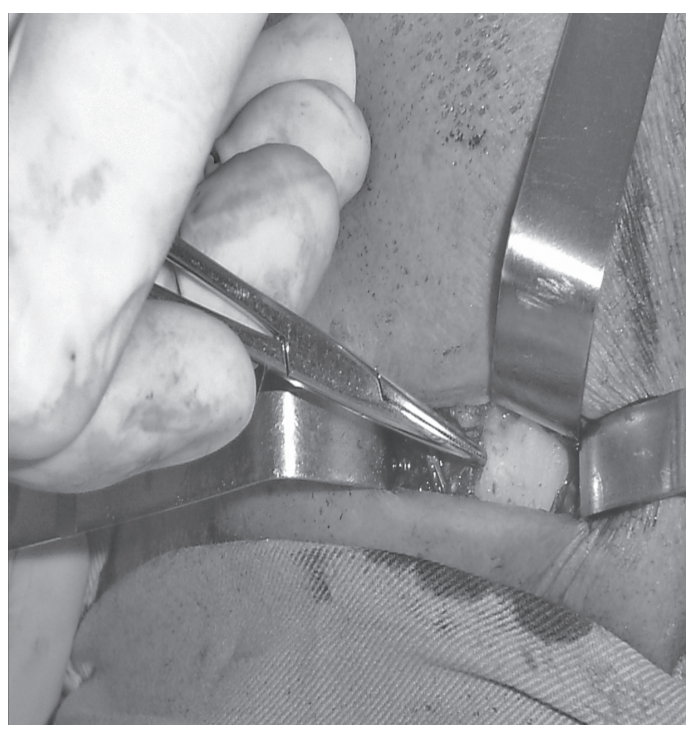

Figura 1 - Incisão sobre a clavícula

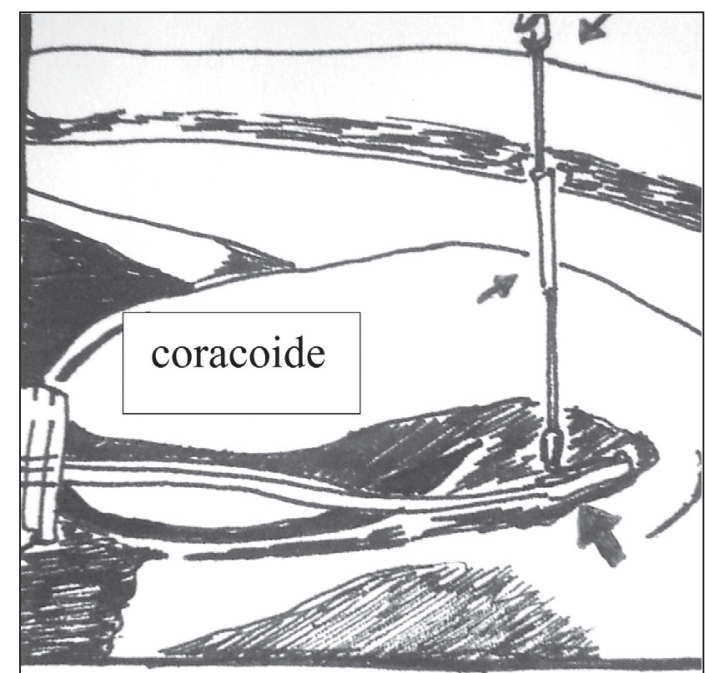

Figura 2 - confecção do túneis ósseos

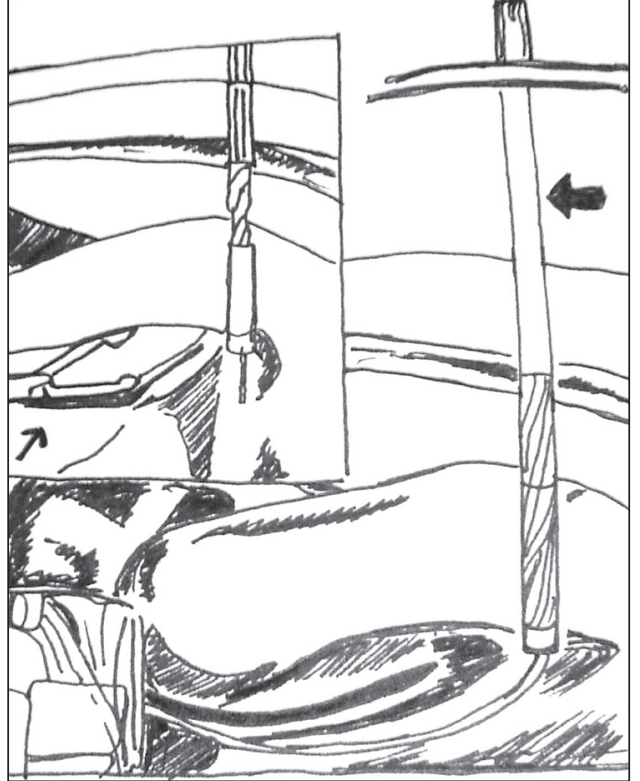

Figura 3 - Captura do fio-guia maleável

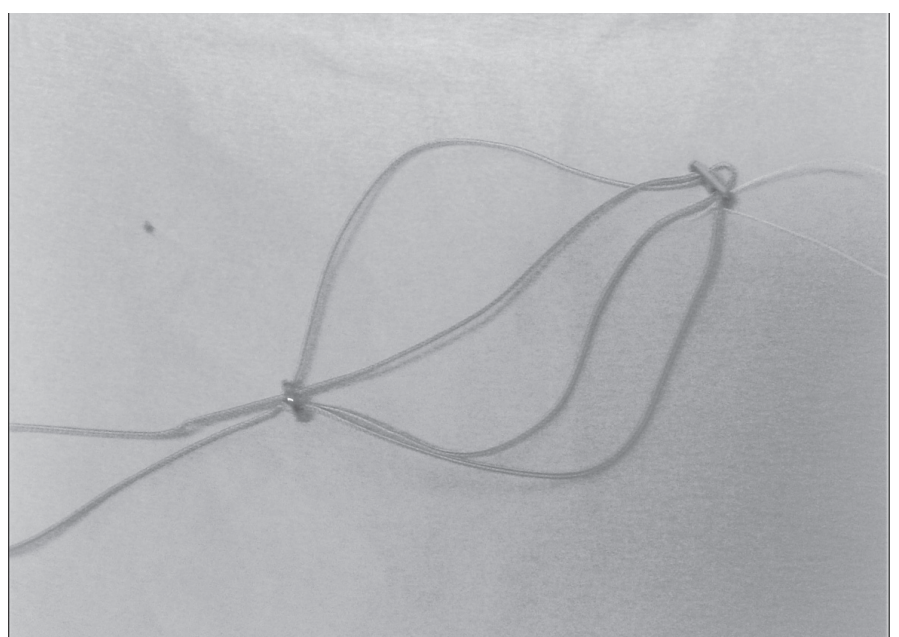

Figura 4 - Sistema Tight Rope-Arthrex ${ }^{\circledR}$

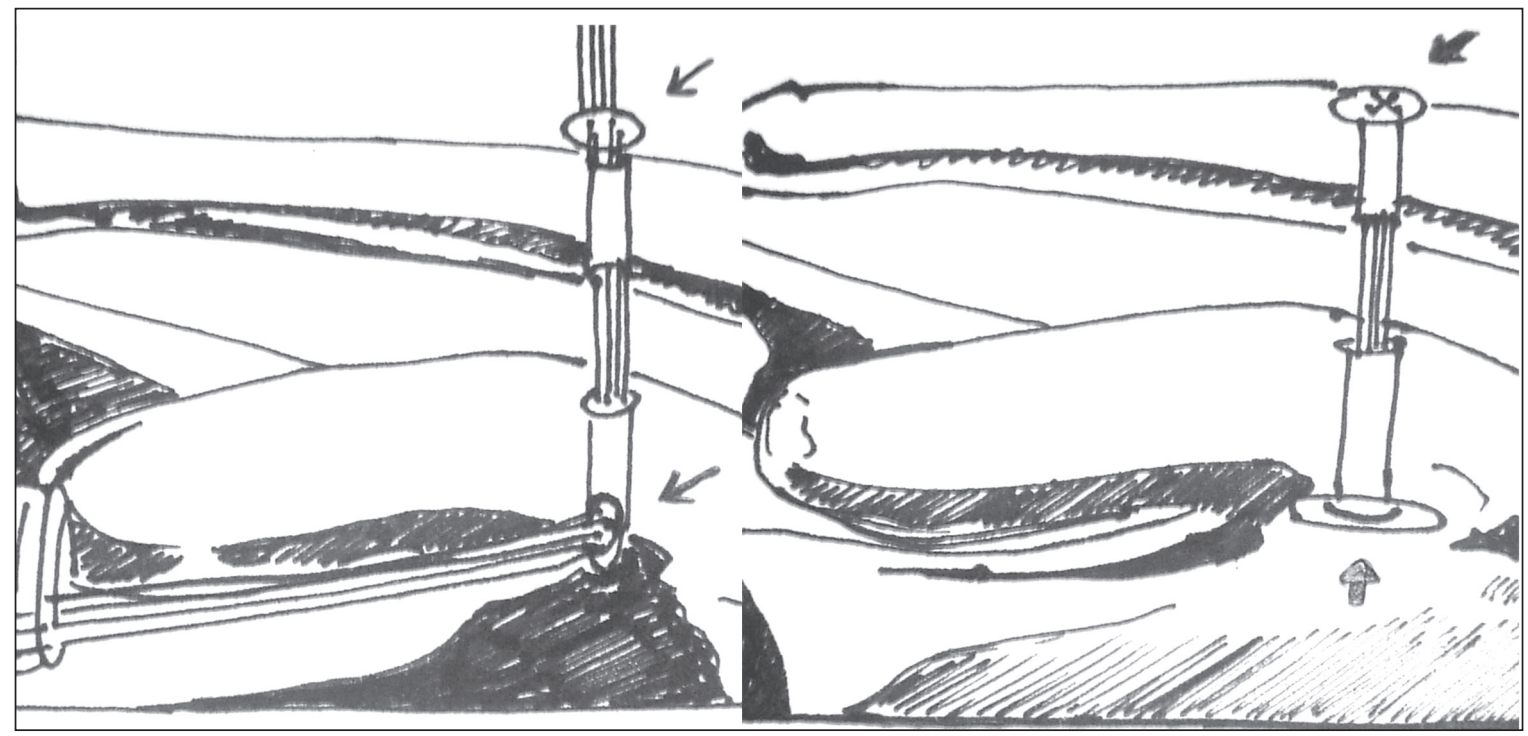

Figuras 5 e 6 - Passagem do sistema e fixação do mesmo após redução articular 


\section{RESULTADOS}

O seguimento pós-operatório médio foi de 15 meses, variando de 12 a 19 meses (Tabela 1).

Utilizando o método de avaliação da escala $\operatorname{UCLA}^{(12)}$, observamos bons e excelentes resultados em todos os casos com média de 32,5 pontos (Tabela 1 ).

Após uma semana pós-operatória, um dos pacientes sofreu nova queda sobre o ombro operado e não sofreu nenhum comprometimento do sistema implantado com a articulação acrômio-clavicular mantida reduzida clínica e radiologicamente (Figura 7).

Todos os pacientes operados agudamente encontravam-se satisfeitos com os resultados do tratamento cirúrgico artroscópico (Figuras 8 e 9).

O grau de amplitude de movimento da articulação glenoumeral de todos os pacientes voltou ao mesmo arco de movimento pré-trauma - flexão de $135^{\circ}$ a $140^{\circ}$ e abdução $90^{\circ}$ - conforme informação colhida dos próprios pacientes comparando-se com lado contra-lateral. Aqueles pacientes com UCLA 29 e 30 foram decorrentes de dor residual ao nível da articulação acrômio-clavicular com alguma limitação da amplitude de movimento em função desta dor residual. No entanto, todos informaram estarem satisfeitos com o resultado pós-operatório final quando comparado com o quadro clínico pré-operatório.

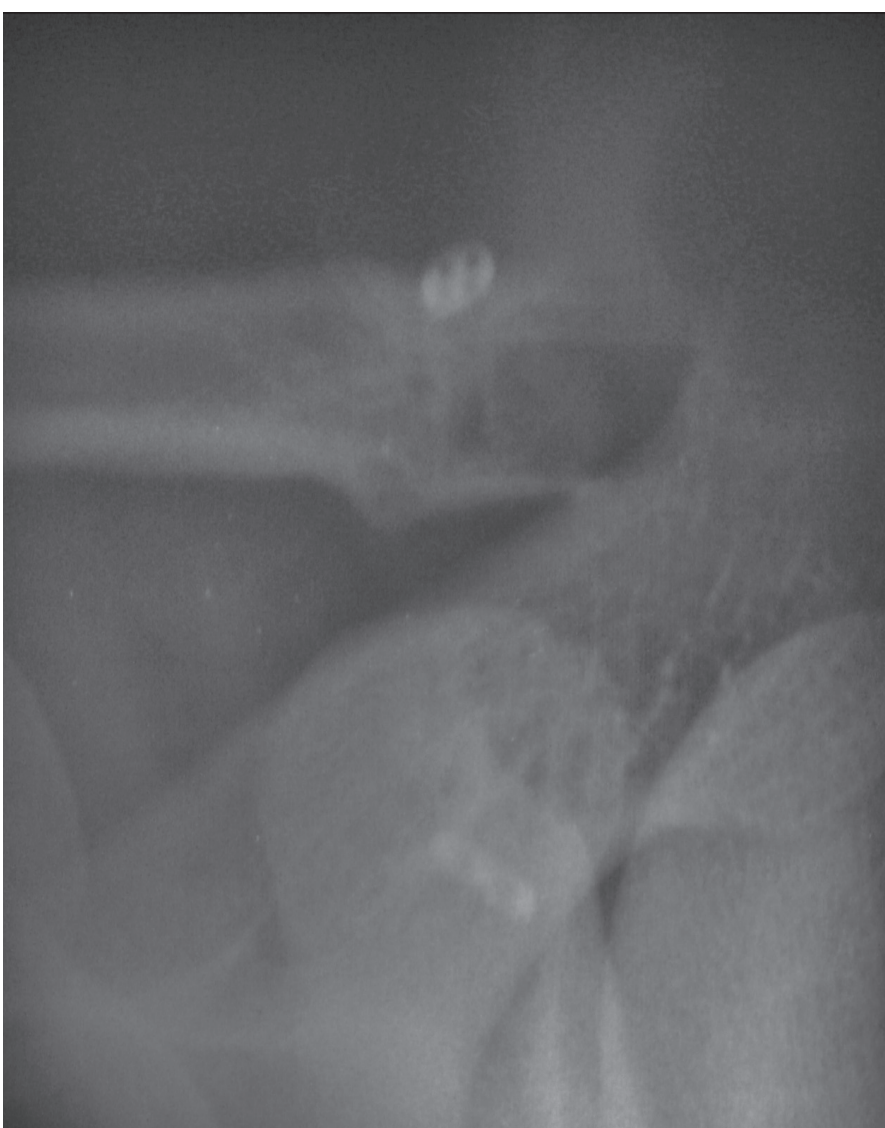

Figura 7 - Raio X simples com sistema Tight rope ${ }^{\circledR}$ implantado e articulação acrômio-clavicular reduzida
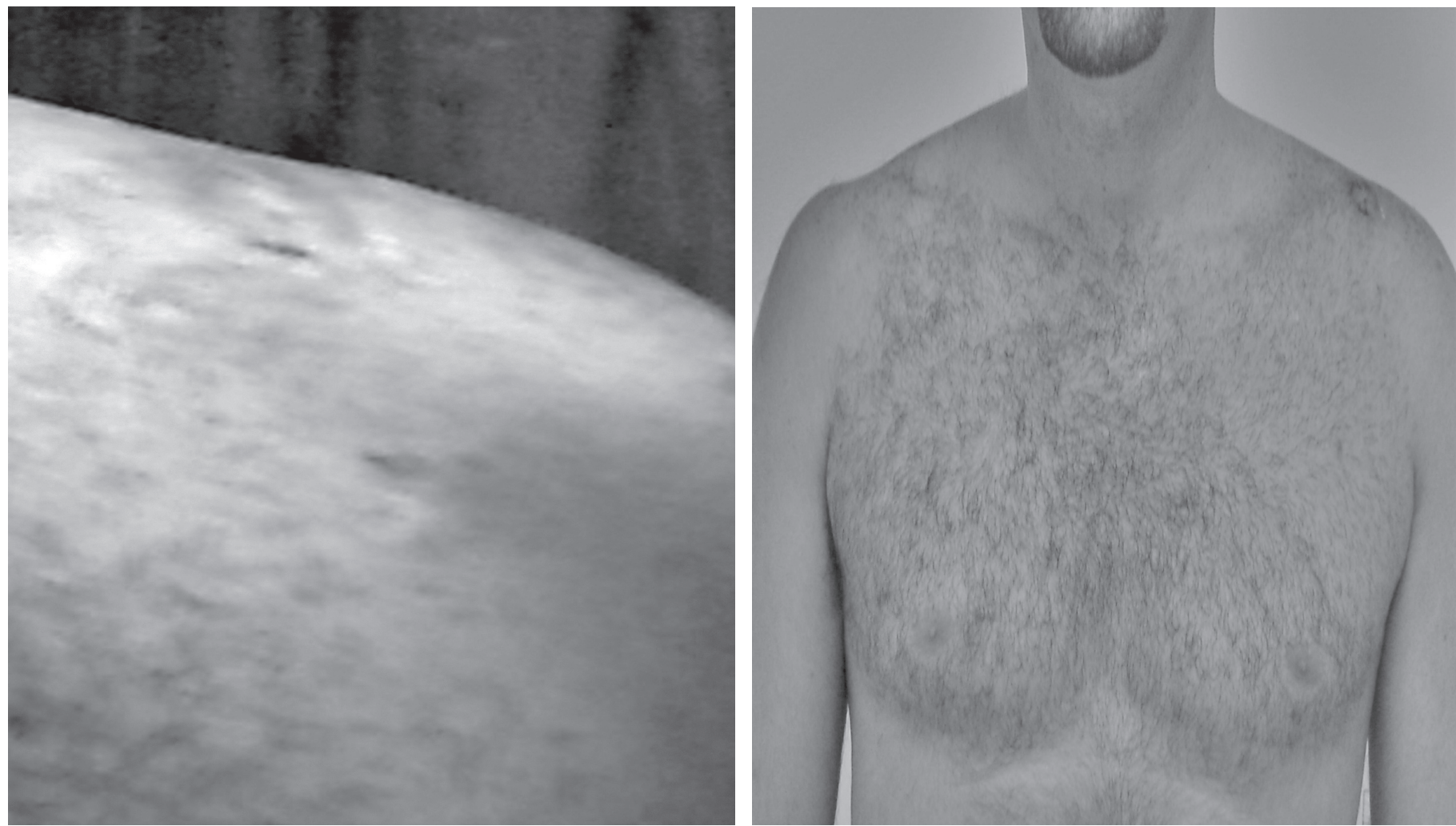

Figuras 8 e 9 - Aspecto clínico após implantação do sistema Tight Rope ${ }^{\circledR}$ com articulação acrômio-clavicular reduzida 


\section{DISCUSSÃO}

O tratamento da luxação acrômio-clavicular tipos I e II é conduzido conservadoramente com a maioria dos pacientes retornando às condições pré-lesão ${ }^{(3-7)}$ enquanto que os tipos IV, V e VI são tipicamente tratados cirurgicamente $^{(8-12)}$. Embora o tratamento da LAC tipo III seja controverso ${ }^{(13)}$, muitos recomendam o tratamento conservador com o uso de tipóia simples não se preocupando com a redução da luxação ${ }^{(14)}$ enquanto outros acreditam na necessidade de cirurgia ${ }^{(15)}$. Nós observamos melhores resultados quando tratamos cirurgicamente estes pacientes através de avaliação clínica subjetiva e por aferimento de grau de amplitude de movimento e força muscular por aparelho específico tipo Cybex e, portanto, defendemos esta última opção.

O tratamento cirúrgico da luxação acrômio-clavicular é tipicamente reservado para os deslocamentos completos. Nas luxações agudas da articulação acrômio-clavicular, a reconstrução dos ligamentos coracoclaviculares, também, tem sido empregado ${ }^{(16-18)}$. As opções cirúrgicas são inúmeras podendo o cirurgião escolher entre dezenas de técnicas ${ }^{(11)}$. Nós, atualmente, optamos pelo sistema Tight Rope - Arthrex ${ }^{\circledR}$ que busca substituir os ligamentos coracoclaviculares através dos quatro fios Fiberwire - Arthrex ${ }^{\circledR}$ № 5 de forma que são suportados em tensão através do botões metálicos supraclavicular e infracora-

\section{REFERÊNCIAS}

1. Mazzocca AD, Santangelo SA, Johnson ST, Rios CG, Dumonski ML, Arciero RA. A biomechanical evaluation of an anatomical coracoclavicular ligament reconstruction. Am J Sports Med. 2006;34(2):236-46.

2. Rockwood CA Jr, Williams GR, Young DC. Disorders of the acromioclavicular joint. In: Rockwood CA, Matsen FA, editors. The shoulder. 2nd ed. Philadelphia: Saunders; 1998. p. 483-553.

3. Cox JS. The fate of the acromioclavicular joint in athletic injuries. Am J Sports Med. 1981;9:50-3.

4. Dias JJ, Steingold RF, Richardson RA, Tesfayohannes B, Gregg PJ. The conservative treatment of acromioclavicular joint dislocation: review after five years. J Bone Joint Surg Br. 1987;69:719-22.

5. Galpin RD, Haekins RJ, Grainger RW. A comparative analysis of operative versus non-operative treatment of grade III acromioclavicular separations. Clin Orthop Relat Res. 1985;193:150-55.

6. Lemos MJ. The evaluation and treatment of the injured acromioclavicular joint in athletes. Am J Sports Med. 1998;26:137-44.

7. Bannister GC, Wallace WA, Stableforth PG, Hutson MA. The management of acute acromioclavicular dislocation: a randomized prospective controlled trial. J Bone Joint Surg Br. 1989;71:848-50.

8. Larsen E, Berg-Nielsen A, Christensen P. Conservative or surgical treatment of acromioclavicular dislocation: a prospective, controlled, randomized study. J Bone Joint Surg Am. 1986;68:552-5.

9. Sloan SM, Budoff JE, Hipp JA, Nguyen L. Coracoclavicular ligament reconstruction using the lateral half of the conjoined tendon. $J$ Shoulder Elbow Surg. 2004;13:186-90.

10. Tienen TG, Oyen JF, Eggen P. A modified technique of reconstruction for complete acromioclavicular dislocation: a prospective study. Am J Sports Med. 2003;31:655-9. cóide por meio de visão sob artroscopia. Ao nosso conhecimento a primeira experiência compartilhada utilizando esta técnica artroscópica foi publicada em $2006^{(19)}$.

A literatura confirma a eficácia do sistema Tight Rope - Arthrex ${ }^{\circledR}$ com resultados semelhantes aos nossos o que vem a reforçar a eficácia do método e assim incentivar a aplicação deste procedimento minimamente invasivo $^{(19,20)}$

Veado et al chamam a atenção para a não realização da cirurgia na fase aguda quando houver escoriação/ abrasão no local ou presença de flictenas ou infecção cutânea na região da cintura escapular ${ }^{(16)}$. Conduta esta também por nós defendida que mesmo aplicando um procedimento minimamente invasivo, entendemos que é um risco desnecessário comprometer a cirurgia sem corrigir antes tais problemas.

\section{CONCLUSÃO}

A aplicação do sistema Tight Rope - Arthrex ${ }^{\circledR}$ no tratamento artroscópico da luxação acrômio-clavicular mostrou-se técnica de menor agressão cirúrgica, eficiente na promoção do alívio da dor com correção da deformidade e recuperação do arco de movimento em curto espaço de tempo.

Trata-se de um procedimento de baixa morbidade e de fácil aplicação após domínio da técnica.

11. Weaver JK, Dunn HK. Treatment of acromioclavicular injuries, especially complete acromioclavicular separation. J Bone Joint Surg Am. 1972;54:1187-94.

12. Ellman H, Hanker G, Bayer M. Repair of the rotator cuff. End-result study of factors influencing reconstruction. J Bone Joint Surg Am. 1986;68(8):1136-44.

13. Bailey RW. A dynamic repair for complete acromioclavicular joint dislocation. J Bone Joint Surg Am. 1965;47:858

14. Veado MAC, Paiva AA, Pinto MS. Tratamento cirúrgico da luxação acromioclavicular completa. Rev Bras Ortop. 2000;35(8):309-13.

15. Galpin RD, Hawkins RJ, Grainer RW. A comparative analysis of operative versus nonoperative treatment of grade III acromioclavicular reparations. Clin Orthop Relat Res. 1985;193:150-5.

16. Harris RI, Wallace AL, Harper GD, Goldberg JA, Sonnabend DH Walsh WR. Structural properties of the intact and the reconstructed coracoclavicular ligament complex. Am J Sports Med. 2000;28:103-8.

17. Tsou PM. Percutaneous cannulated screw coracoclavicular fixation for acute acromioclavicular dislocations. Clin Orthop Relat Res. 1989;243:112-21

18. Weinstein DM, McCann PD, Mcllveen SJ, Flatow EL, Bigliani LU. Surgical treatment of complete acromioclavicular dislocations. Am J Sports Med. 1995;23:324-31.

19. Hernegger GS, Kadletz R. Tight rope - the revolutionary anatomical fixation in acromioclavicular joint dislocation - a case report. Tech Shoulder Elbow Surg. 2006;7(2):86-8.

20. Richards A, Duncan TT. Arthroscopic stabilization of acute acromioclavicular joint dislocation using the tightrope system. Tech Shoulder Elbow Surg. 2008;9(2):51-4. 